\title{
Changes in synovial fluid biomarkers after experimental equine osteoarthritis
}

\author{
Tian-wen Ma, Yue Li, Guan-ying Wang, Xin-ran Li, Ren-li Jiang, \\ Xiao-peng Song, Zhi-heng Zhang, Hui Bai, Xin Li, Li Gao \\ College of Veterinary Medicine, \\ Northeast Agricultural University, Harbin 150000, China \\ gaoli43450@163.com
}

Received: July 5, $2017 \quad$ Accepted: November 9, 2017

\begin{abstract}
Introduction: The study aimed to clarify the changes in the concentration of inflammatory mediators, proteases, and cartilage degradation biomarkers in the synovial fluid of joints in an equine osteoarthritis model. Material and Methods: Osteoarthritis was induced in eight Mongolian horses by a sterile intra-articular injection of amphotericin B, which was injected into the left carpal joint in a dose of $2 \mathrm{~mL}(25 \mathrm{mg} / \mathrm{mL})$. The control group comprised five horses which were injected with an equal dose of sterile physiological saline into the left carpal joint. Synovial fluid was obtained at baseline and every week after injection. Test methods were based on ELISA. Results: In the course of the osteoarthritis, the concentration of biomarkers in joint synovial fluid showed an increasing trend. IL-1, IL-6, MMP-9, MMP-13, ADAMTS-5, CS846, GAG, HA, CTX- II, and COMP concentrations sharply increased before the onset of significant symptoms of lameness, whereas TNF- $\alpha$, MMP-2, and MMP-3 concentrations rose sharply after the occurrence of such symptoms. Conclusion: The results obtained confirm that the concentrations of IL-1, IL-6, MMP-9, MMP-13, ADAMTS-5, CS846, GAG, HA, CTX- II and COMP increase substantially in equine osteoarthritis, which provides a theoretical basis for the rapid diagnosis of the disease.
\end{abstract}

Keywords: horse, osteoarthritis, biomarkers, synovial fluid.

\section{Introduction}

Lameness which results from osteoarthritis (OA) is a major reason for early retirement of horses. The diagnosis of $\mathrm{OA}$ relies on clinical and radiographic examination in the late irreversible stages. Sensitive serum or synovial fluid (SF) biomarkers specific for early, potentially reversible, stages of the disease have not been definitively established (12). In the process of equine OA pathogenesis, articular cartilage lesions are the main symptom and produce the inflammatory mediators, protein enzymes, and degradation products of the metabolism of cartilage, which are secreted into the synovial fluid. The SF biomarkers can be detected at an early stage of $\mathrm{OA}$, before the presence of radiographic signs (23). These biomarkers can forecast disease, monitor the response to treatment, and can be used to assess the degree of OA (5).

Proinflammatory factors and tumour necrosis factor $(\mathrm{TNF}-\alpha)$ promote the secretion of proteases and cartilage degradation, which eventually results in the degradation of the extracellular matrix (ECM) (19). Interleukin 1 (IL-1) has been shown to play a pivotal role in cartilage matrix degradation in that it can stimulate chondrocytes to induce the expression of matrix metalloproteinases (MMPs), a disintegrin and metalloproteinase with thrombospondin motifs (ADAMTS), and other catabolic enzymes (29). Interleukin 6 (IL-6) is believed to be one of the major factors in joint destruction, being a pleiotropic proinflammatory cytokine which is markedly upregulated at times of tissue inflammation (22). Inflammatory factors can promote the expression of ADAMTS-5 and MMPs in cartilage cells and accelerate the damage of cartilage.

Aggrecanase-mediated aggrecan degradation is a significant event in the early stage of OA (27). Collagenase MMP-13 can degrade fibrinous collagen (collagens I, II, and III), stromelysin MMP-3 can degrade proteoglycan and non-helical regions of collagen, and gelatinase MMP-2 and MMP-9 can degrade the special types of collagen (collagens I, IV, 
V, and VI) (18). C-terminal crosslinked telopeptide type II collagen (CTX-II) is one of the primary products of type II collagen degeneration. Studies have shown that the activation of MMP-9 was associated with an increase in the concentration of MMP-2 in the inflammatory pathology of equine joint disease (25). Cartilage oligomeric matrix protein (COMP), which is a tissue-specific protein that binds to type II collagen network. When the articular cartilage is destroyed, COMP is released into the SF and absorbed by the serum (2). Chondroitin sulphate 846 (CS846) is a CS synthetic marker and inseparable from the degree of joint injury in patients with osteoarthritis. CS846 and glycosaminoglycan (GAG) are biomarkers of proteoglycan degradation in the cartilage matrix $(6,7)$. Hyaluronic acid is a connexin of collagen and proteoglycan in the cartilage matrix and present as a coat around each chondrocyte (27). Currently, none of these SF biomarkers are used in veterinarian clinical practice for the detection of equine $\mathrm{OA}$ at an early stage. The overall objective of this study was to examine the changes in SF biomarkers after experimental equine OA. The results from this study will provide a better understanding of the molecular changes and tissue damage occurring in the cartilage in the equine OA process.

\section{Material and Methods}

A total of 13 healthy 5 to 8-year-old mixed-breed Mongolian male and female horses were used. Their body weight was between $331 \mathrm{~kg}$ and $511 \mathrm{~kg}$. Prior to being admitted into the study, assessments of body condition scores, response to carpal flexion, and synovial effusion, were performed as were carpal radiography and an examination for lameness to ensure all measured variables were within reference ranges. Horses were allowed a 2 -week acclimatisation period with once-daily hand-walking and were box-rested in separate $4.0 \times 4.0 \mathrm{~m}^{2}$ stalls on woodchip bedding for the duration of the experiment.

The horses were randomly assigned into the experimental group $(n=8)$ or control group $(n=5)$, adjusted according to gender and age. At baseline, animals were sedated intravenously with $10 \mu \mathrm{g} / \mathrm{kg}$ of detomidine hydrochloride (Sigma-Aldrich, USA) and $0.1 \mathrm{mg} / \mathrm{kg}$ of methadone hydrochloride (SigmaAldrich, USA). Aseptic arthritis was induced in horses in the experimental group on day 0 using $25 \mathrm{mg} / \mathrm{mL}$ of amphotericin B (catalogue no. 1633C219, AmrescoSolon, USA) in $2 \mathrm{~mL}$ of sterile water as a single intraarticular injection in the left carpal joint. The left carpal joint of control horses was injected with isopyknic sterile physiological saline solution (catalogue no. 160630D9, San Lian Pharm-Harbin, China). The horses did not receive any anti-inflammatory drug which could interfere with the development of OA. SF $(0.5-1 \mathrm{~mL})$ was collected through aseptic arthrocentesis from the joint before amphotericin $\mathrm{B}$ administration (T0). The SF samples were collected weekly throughout the experiment until the ninth week. Samples were collected in Eppendorf tubes without additives. Collected samples were centrifuged at $10,000 \times \mathrm{g}$ for $15 \mathrm{~min}$ within $1 \mathrm{~h}$. Supernatant was stored at $-80^{\circ} \mathrm{C}$ until future analysis. After each SF collection, rectal temperature, respiratory rate, pulse, and circumference of the carpal joint were recorded. Furthermore, lameness at trot was assessed once a week and scored using the American Association of Equine Practitioners (AAEP) grading scale (Table 1) (21). All clinical indicators were measured and evaluated by the same observer.

Table 1. The claudication grading of horses

\begin{tabular}{ll}
\hline Grade & Clinical symptoms \\
\hline 0 & Sound \\
\hline 1 & $\begin{array}{l}\text { Lameness difficult to detect and inconsistent. In certain } \\
\text { circumstances (such as weight carrying, circling, } \\
\text { inclines, hard surfaces) apparent intermittent } \\
\text { claudication }\end{array}$ \\
\hline 2 & $\begin{array}{l}\text { Lameness difficult to detect, but consistent. In certain } \\
\text { circumstances (such as weight carrying, circling, } \\
\text { inclines, hard surfaces) apparent sustained claudication }\end{array}$ \\
\hline 3 & $\begin{array}{l}\text { Lameness is consistently observable at a trot under all } \\
\text { circumstances }\end{array}$ \\
\hline 5 & $\begin{array}{l}\text { Obvious lameness with marked head nodding, or } \\
\text { shortened stride }\end{array}$ \\
\hline
\end{tabular}

Concentrations of IL-1, IL-6, TNF- $\alpha$, ADAMTS-5, MMP-2, MMP-3, MMP-9, MMP-13, CS846, GAG, CTX-II, COMP, and hyaluronic acid (HA) were determined using a commercially available ELISA kits (Thermo Fisher Scientific, USA). All measurements were taken according to the manufacturer's instructions. The linear regression curve of the kit standard was drawn and the OD value was brought into the equation to calculate the concentration of each sample.

A software package (SPPS version 18.0 for Windows, SPPS, USA) was used for all analyses. Data were expressed as the mean \pm standard error. Statistical analyses were undertaken by repeated measures ANOVA and the Student's $t$-test for repeated measures. The significance level was set at $\mathrm{P}<0.05$.

\section{Results}

All horses had no lameness before injection of amphotericin B and other clinical signs were normal. As was expected, amphotericin B injection resulted in a remarkable increase in the circumference of carpal joints (Fig. 1). The horses showed grade 1 lameness in the first two weeks after the establishment of the osteoarthritis model. In the third to fifth weeks they showed grade 2 lameness, while grade 3 lameness was shown in the sixth to eighth weeks and grade 5 lameness in the ninth week. Injections of sterile 
physiological saline solution into the left carpal joints did not lead to lameness.

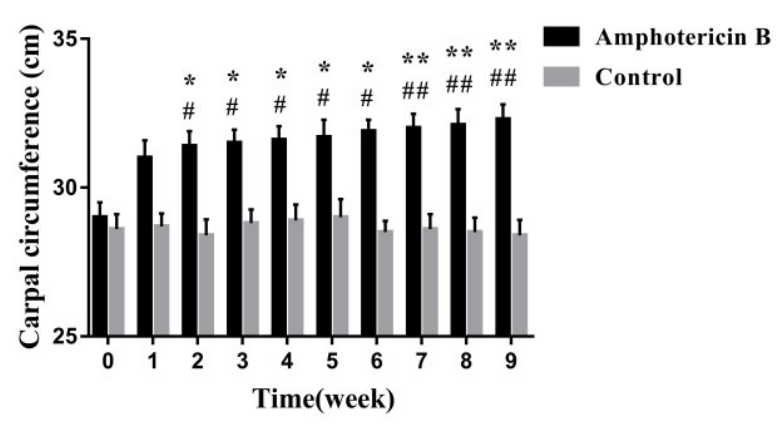

Fig. 1. Clinical results after amphotericin B induced OA. Carpal circumference following induction of acute synovitis in the middle of carpal joints of horses $(n=13)$. Data are shown as the mean \pm standard error of the mean. $* \mathrm{P}<0.05$ or $* * \mathrm{P}<0.01$ compared with baseline ( $\mathrm{t}=0) ;{ }^{\#} \mathrm{P}<0.05$ or ${ }^{\#} \mathrm{P}<0.01$ compared with control group

Compared with the control group, both IL-1 and IL-6 concentrations in the experimental group increased sharply from week 1 to week $9(\mathrm{P}<0.05)$. The TNF- $\alpha$ concentration increased after the first week of model establishment. The concentration of TNF- $\alpha$ was significantly increased from the third week to the ninth week after injection of amphotericin $(\mathrm{P}<0.05)$ (Fig. 2).

The concentrations of MMP-2, MMP-3, MMP-9, MMP-13, and ADAMTS-5 increased continuously over nine weeks after amphotericin B injection. Compared with the control joint, MMP-2 and MMP-3 increased significantly from the third to the ninth week $(\mathrm{P}<0.05)$, ADAMTS-5 and MMP-9 increased significantly from the first to the ninth week $(\mathrm{P}<0.05)$, and MMP-13 increased rapidly from the second to the ninth week $(\mathrm{P}<0.05)$ (Fig. 3).

Compared with the control group, the concentrations of CS846, GAG, and HA rose with the passage of time and very significant differences were demonstrated from the first week to the ninth week $(\mathrm{P}<0.05)$. From the second to ninth week following amphotericin B injection, the concentrations of COMP and CTX-II in synovial fluid increased significantly $(\mathrm{P}<0.05)$ (Fig. 4).

In the control group, none of the biomarkers changed significantly.
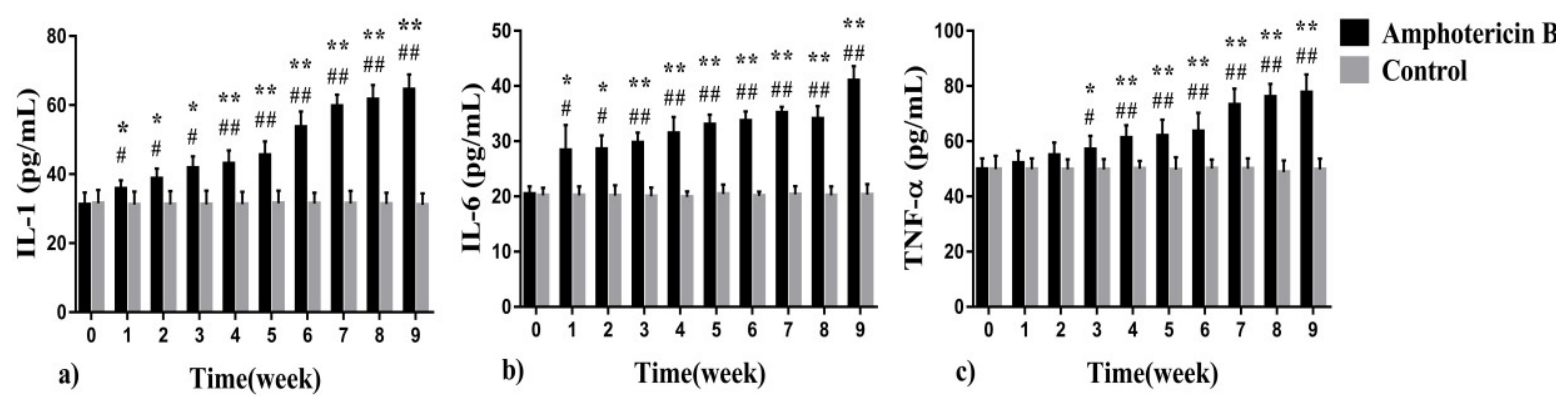

Fig. 2. Synovial fluid inflammatory biomarkers in amphotericin B-treated and control joints. (a) interleukin 1 (IL-1), (b) interleukin 6 (IL-6), and (c) tumour necrosis factor- $\alpha(\mathrm{TNF}-\alpha)$ over time in inflamed model and control joints of horses $(\mathrm{n}=13)$. Data are shown as the mean \pm standard error of the mean. ${ }^{*} \mathrm{P}<0.05$ or ${ }^{* *} \mathrm{P}<0.01$ compared with baseline $(\mathrm{t}=0) ;{ }^{\#} \mathrm{P}<0.05$ or ${ }^{\# \#} \mathrm{P}<0.01$ compared with control group
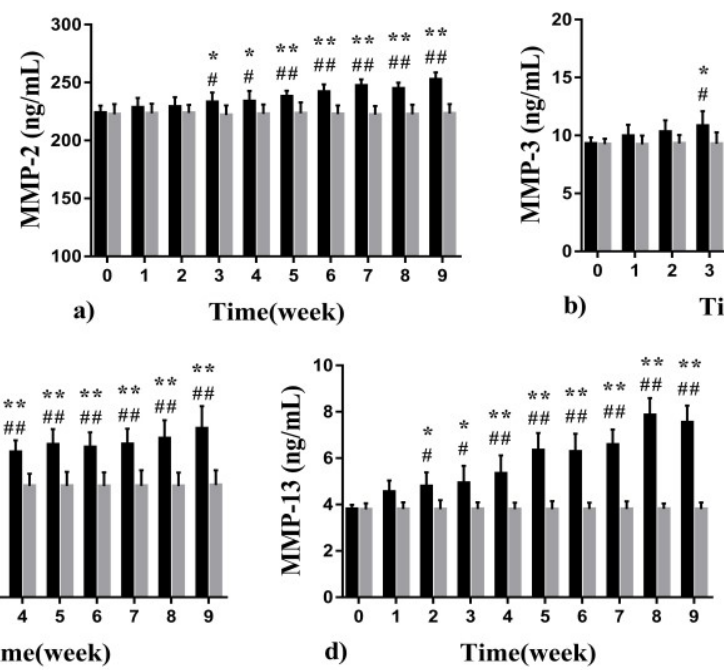

Amphotericin B

Control
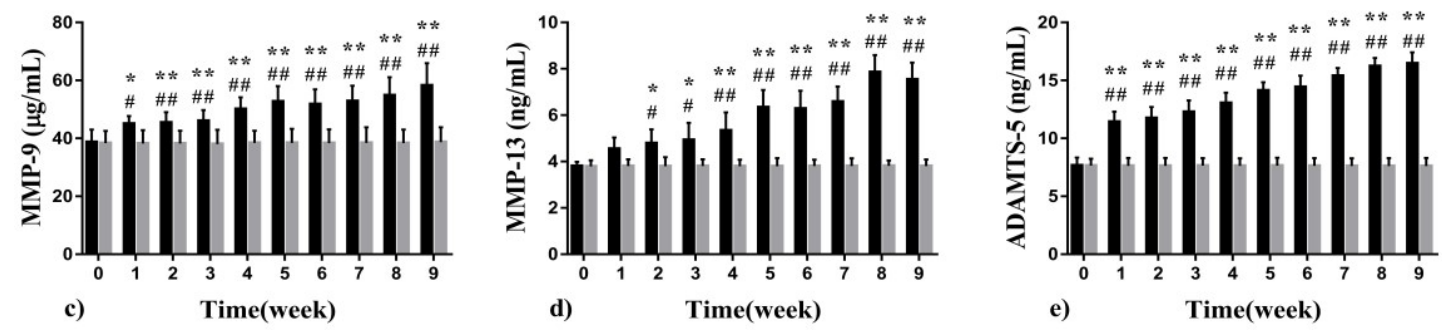

Fig. 3. Synovial fluid proteases in amphotericin B treated and control joints. (a) MMP-2, (b) MMP-3, (c) MMP-9, (d) MMP-13, (e) ADAMTS-5 over time in inflamed model and control joints of horses $(n=13)$. Data are shown as the mean \pm standard error of the mean. ${ }^{*} \mathrm{P}<0.05$ or ${ }^{* *} \mathrm{P}<0.01$ compared with baseline $(\mathrm{t}=0) ;{ }^{*} \mathrm{P}<0.05$ or ${ }^{\# \#} \mathrm{P}<0.01$ compared with control group 

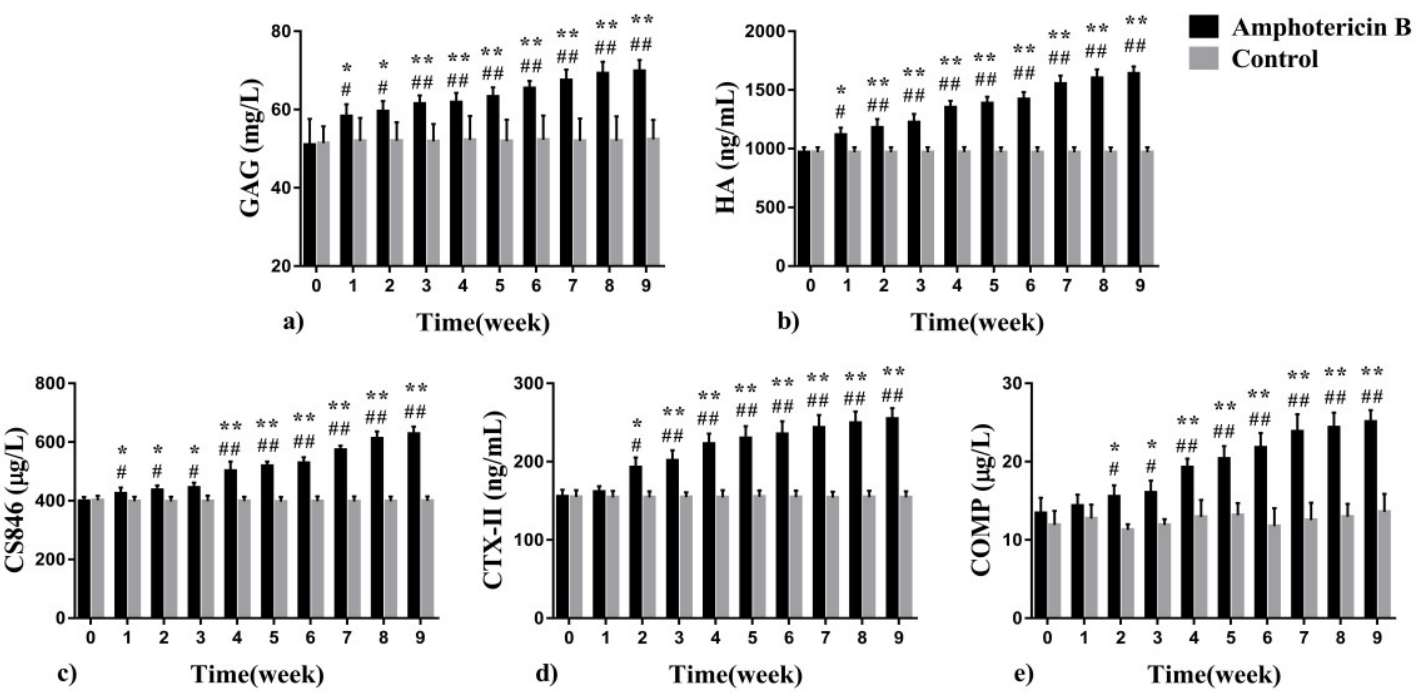

Fig. 4. Synovial fluid concentrations of cartilage degradation biomarkers in amphotericin $\mathrm{B}$ and control joints. (a) glycosaminoglycan (GAG), (b) hyaluronic acid (HA), (c) chondroitin sulphate 846 (CS846), (d) C-terminal crosslinked telopeptide type II collagen (CTX-II), and (e) cartilage oligomeric matrix protein (COMP) over time in inflamed model and control joints of horses $(\mathrm{n}=13)$. Data are shown as the mean \pm standard error of the mean. $* \mathrm{P}<0.05$ or $* * \mathrm{P}<0.01$ compared with baseline $(\mathrm{t}=0) ;{ }^{\#} \mathrm{P}<0.05$ or ${ }^{\#} \mathrm{P}<0.01$ compared with control group

\section{Discussion}

In the past few years, the importance of SF biomarkers in equine osteoarthritis, joint effusion, synovitis, and progression of cartilage damage has become increasingly appreciated as useful for monitoring disease progression or clinical treatment (6). However, serious notice of the magnitude and timing of the effects of equine OA on SF marker concentrations has been taken by relatively few. In the present study, the reason that we used the OA model (amphotericin B) was its being a well-accepted model for inducing equine $\mathrm{OA}(3,20)$. In the OA model in the donkey established by Mokbel et al. (21), mild symptoms occurred after three weeks, moderate symptoms six weeks later, and severe symptoms at the ninth week. The horse model is a great translational model for estimating intra-articular inflammation with outcomes relevant to human OA.

Analysis of inflammatory mediators in SF indicates that IL-1 and IL-6 levels increased significantly from the first week after the establishment of the equine OA model, before any significant limp. The results showed that the inflammatory response occurred in the joints after inducing the osteoarthritis model. The pathways of inflammatory factors were generated in synovial cells and chondrocytes, and the levels of IL-1 and IL-6 secreted continued to increase. Chondrocytes and synovial cells were stimulated to release increased amounts of ADAMTS and MMPs such as collagenases and stromelysin, and promoted cartilage degradation. The amount of IL- 6 produced by healthy chondrocytes is small, and this amount has little effect on joint tissue. However, when chondrocytes are in an inflammatory environment, IL-6 can inhibit type II collagen in cartilage matrix form (17). Previous studies have shown that the addition of anti-TNF- $\alpha$ antibodies to the synovial membrane can inhibit the production of IL-1 and IL-6 (13). In equine OA, TNF- $\alpha$ expression was significantly increased (16). These conclusions indicate that TNF- $\alpha$, IL-1, and IL-6 have an inevitable link, and these factors promote the development of osteoarthritis. However, levels of TNF- $\alpha$ were not correlated with the degree of joint damage in a study by Joughlin et al. (14). This is an important observation, as the role of TNF- $\alpha$ in articular cartilage degradation is controversial. We hypothesise that they all contribute to cartilage turnover. The present study helps elucidate the long effect of equine OA on these inflammatory mediators in vivo.

Production of MMPs and ADAMTSs is known to be induced by inflammatory stimuli, such as IL-1 and TNF- $\alpha$. In the pathogenesis of horse joint disease, the activation of MMP-9 is associated with an increase in MMP-2 concentration (9). Reportedly, MMP-9 is produced and released into the SF by chondrocytes and synoviocytes in diseased joints in horses but not in clinically normal joints (4). In our study, latent and activated MMP-9 was present in SF from all equine joints. Similar findings have been reported in horses by Gaudin et al. (10). It has been shown that a significant increase in MMP-2 content occurs after significant lameness. Under the action of various inflammatory factors, a large amount of MMP-9 is produced in SF earlier than MMP-2. Stromelysin MMP-3 degrades collagen types II, IV, and IX, and proteoglycans, and also promotes the activation of MMP-1 and MMP-13. The MMP-3 in the model group increased significantly from the third week, and the time of production of MMP-3 was the same as that of significant lameness. In 
the animal model of joint disease, changes in the content of MMP-3 in SF were relatively rare (9). The concentration of MMP-13 in the SF increased rapidly from the second week after intra-articular injection of amphotericin B. This is due to the increase in the level of MMP-13 in the tissue by the stimulation of inflammatory mediators, resulting in an increase in the amount of MMP-13 secreted into SF. ADAMTS-5 plays a significant role in the degradation process (21). Compared to the control group, ADAMTS-5 was significantly increased from the first week to the end of the trial. The expression of ADAMTS-5 is also affected by inflammatory factors, and the cytokines in inflammation can promote the expression of ADAMTS-5 in cartilage (22). This result again demonstrated that the degradation of proteoglycans occurred in the early events of OA.

With the development of the inflammation in the joints, the inflammatory factors and proteases produced by joint tissue gradually proliferated, the degradation of articular cartilage gradually increased, and individual direct biomarkers of cartilage metabolism were secreted into synovial fluid. CS846 and GAG are biomarkers of proteoglycan in cartilage matrix. Remarkably high levels of GAGs were found in synovial fluid samples from horses with traumatic arthritis and osteochondritis dissecans (OCD) (1). In another study, because of a lack of correlation between GAG concentration and CDI or MMP activity in metacarpophalangeal joints collected post mortem, the usefulness of this marker for monitoring equine joint disease is suggested to be severely limited (26). However, the concentration of CS846 and GAG in the SF began to increase significantly from the first week after the model was established. This result suggests that cartilage degradation occurs under the action of inflammatory factors and proteases, and the time of degradation of proteoglycans is earlier. CTX-II is considered a marker of collagen II decomposition. CTX-II plays a fundamental role in assessing the extent of cartilage damage. The study illustrated that in the model group horses it increased sharply after the second week compared with the control group. A significant increase in CTX-II concentration occurred before significant lameness, which was consistent with the previous research (28). The degradation of type II collagen occurred late, which may be related to the time of action of MMP-13 and the complex helical structure of type II collagen. The relationship between HA concentration and cartilage injury is controversial. It has been shown that there is no correlation between $\mathrm{HA}$ in the SF and cartilage injury (8). In this study, we find that the change of HA content in the SF was positively correlated with the injury of articular cartilage. After the establishment of the model, the amount of COMP in horse SF began to increase significantly from the second week, and gradually increased with the development of OA. One related study concluded that an elevated synovial fluid
COMP concentration could be a useful marker for diagnosis and monitoring of equine middle carpal joint fractures in thoroughbreds (24). Also, an increase in synovial fluid COMP concentration correlated with time of post OA injury, suggesting that COMP may be a suitable marker for longitudinal studies to evaluate its role in OA joint injury.

In conclusion, in the course of osteoarthritis, the concentration of biomarkers (IL-1, IL-6, TNF- $\alpha$, ADAMTS-5, MMP-2, MMP-3, MMP-9, MMP-13, CS846, GAG, CTX-II, COMP, and HA) in joint synovial fluid showed an increasing trend. IL-1, IL-6, MMP-9, MMP-13, ADAMTS-5, CS846, GAG, HA, CTX-II, and COMP concentrations sharply increased before significant lameness occurred. We hypothesised that IL-1, IL-6, MMP-9, MMP-13, ADAMTS-5, CS846, GAG, HA, CTX- II, and COMP alone could serve as biomarkers for equine OA. The model presented in this paper will be useful in establishing the therapy of equine joint disease through identifying changes in SF biomarkers.

Conflict of Interests Statement: The authors declare that there is no conflict of interests regarding the publication of this article.

Financial Disclosure Statement: This work was financially supported by the National Key Research and Development Program of China (2017YFD 050220).

Animal Rights Statement: The authors declare that the experiments on animals were conducted in accordance with local Ethical Committee laws and regulations as regards care and use of laboratory animals. All procedures were approved by the Local Ethical Commission (Northeast Agricultural University, Harbin, China).

\section{References}

1. Alwan W.H., Carter S.D., Bennett D., Edwards G.B.: Glycosaminoglycans in horses with osteoarthritis. Equine Vet $\mathrm{J}$ 1991, 23, 44-47.

2. Bai B., Li Y.: Combined detection of serum CTX-II and COMP concentrations in osteoarthritis model rabbits: an effective technique for early diagnosis and estimation of disease severity. J Orthopaedic Surg Res 2016, 11, 149.

3. Barrachina L., Remacha A.R., Soler L., García N., Romero A., Vázquez F.J., Vitoria A., Álava M.Á., Lamprave F., Rodellar C.: Acute phase protein haptoglobin as inflammatory marker in serum and synovial fluid in an equine model of arthritis. Vet Immunol Immunopathol 2016, 182, 74-78.

4. Clegg P.D., Coughlan A.R., Riggs C.M., Carter S.D.: Matrix metalloproteinases 2 and 9 in equine synovial fluids. Equine Vet J 1997, 29, 343-348.

5. de Bakker E., Stroobants V., VanDael F., Ryssen B.V., Meyer E.: Canine synovial fluid biomarkers for early detection and monitoring of osteoarthritis. Vet Rec 2017, 180, 328-329.

6. de Grauw J.C., van de Lest C.H.A., van Weeren P.R.: Inflammatory mediators and cartilage biomarkers in synovial 
fluid after a single inflammatory insult: a longitudinal experimental study. Arthritis Res Ther 2009, 11, R35.

7. Frisbie D.D., Al-Sobayil F., Billinghurst R.C., Kawcak C.E., McIlwraith C.W.: Changes in synovial fluid and serum biomarkers with exercise and early osteoarthritis in horses. Osteoarthritis Cartilage 2008, 16, 1196-1204.

8. Fuller C., Barr A.R., Sharif M., Dieppe P.: Cross-sectional comparison of synovial fluid biochemical markers in equine osteoarthritis and the correlation of these markers with articular cartilage damage. Osteoarthritis Cartilage 2001, 9, 49-55.

9. Garner B., Stoker A., Kuroki K., Evans R., Cook C.R., Cook J. Using animal models in osteoarthritis biomarker research. J Knee Surg 2001, 24, 251-264.

10. Gaudin P., Razakaboay M., Surla A., Berthier S., Fauconnier J., Morel F., Phelip X.: A study of metalloproteinases in fifty joint fluid specimens. Rev Rhumat 1997, 64, 375-381.

11. Gendron C., Kashiwagi M., Lim N.H., Enghild J.J., Thøgersen I.B., Hughes C., Caterson B., Nagase H.: Proteolytic activities of human ADAMTS-5: comparative studies with ADAMTS-4. J Biol Chem 2007, 282, 18294-18306.

12. Heinegård D., Saxne T.: The role of the cartilage matrix in osteoarthritis. Nature Rev Rheumatol 2001, 7, 50-56.

13. Iwai K.: Diverse ubiquitin signaling in NF- $\kappa B$ activation. Trends Cell Biol 2012, 22, 355-364.

14. Jouglin M., Robert C., Valette J.P., Gavard F., QuintinColonna F., Denoix J.M.: Metalloproteinases and tumor necrosis factor-alpha activities in synovial fluids of horses: correlation with articular cartilage alterations. Vet Res 2000, 31, 507-515.

15. Kamarainen O.P., Solovieva S., Vehmas T., Luoma K., Riihimaki H., Ala-Kokko L., Mannikko M., Leino-Arjas P.: Common interleukin-6 promoter variants associate with the more severe forms of distal interphalangeal osteoarthritis. Arthritis Res Ther 2008, 10, R21.

16. Kamm J.L., Nixon A.J., Witte T.H.: Cytokine and catabolic enzyme expression in synovium, synovial fluid, and articular cartilage of naturally osteoarthritic equine carpi. Equine Vet $\mathbf{J}$ 2010, 42, 693-699.

17. Kapoor M., Martelpelletier J., Lajeunesse D., Pelletier J.P., Fahmi H.: Role of proinflammatory cytokines in the pathophysiology of osteoarthritis. Prog Modern Biomed 2014, 7, 33-42.

18. Kozaci L.D., Buttle D.J., Hollander A.P.: Degradation of type II collagen, but not proteoglycan, correlates with matrix metalloproteinase activity in cartilage explant cultures. Arthritis Rheumatol 1997, 40, 164-174.

19. Maldonado M., Nam J.: The role of changes in extracellular matrix of cartilage in the presence of inflammation on the pathology of osteoarthritis. Biomed Res Int 2013, 2013, 284873.
20. Marttinen P.H., Raulo S.M., Suominen M.M., Tulamo R.M. Changes in MMP-2 and -9 activity and MMP- 8 reactivity after amphotericin B induced synovitis and treatment with bufexamac. J Vet Med 2006, 53, 311-318.

21. Mokbel A.N., El Tookhy O.S., Shamaa A.A., Rashed L.A., Sabry D., El Sayed A.M.: Homing and reparative effect of intraarticular injection of autologus mesenchymal stem cells in osteoarthritic animal model. BMC Musculoskelet Disord 2011, 12,259

22. Sawaji Y., Hynes J., Vincent T., Saklatvala J.: Fibroblast growth factor 2 inhibits induction of aggrecanase activity in human articular cartilage. Arthritis Rheum 2008, 58, 3498-3509.

23. Skioldebrand E., Ekman S., Mattsson Hulten L., Svala E., Bjorkman K., Lindahl A., Lundqvist A., Onnerfjord P., Sihlbom C., Ruetschi U.: Cartilage oligomeric matrix protein neoepitope in the synovial fluid of horses with acute lameness: A new biomarker for the early stages of osteoarthritis. Equine Vet J 2017, 49, 662-667.

24. Skioldebrand E., Heinegard D., Eloranta M.L., Nilsson G., Dudhia J., Sandgren B., Ekman S.: Enhanced concentration of COMP (cartilage oligomeric matrix protein) in osteochondral fractures from racing Thoroughbreds. J Orthop Res 2005, 23, $156-163$.

25. Trumble T.N., Trotter G.W., Oxford J.R., McIlwraith C.W., Cammarata S., Goodnight J.L., Billinghurst R.C., Frisbie D.D.: Synovial fluid gelatinase concentrations and matrix metalloproteinase and cytokine expression in naturally occurring joint disease in horses. Am J Vet Res 2001, 62, 1467-1477.

26. Boom R., Brama P.A., Geesje H., Kiers G.H., Groot K.J., Weeren P.R.: Assessment of the effects of age and joint disease on hydroxyproline and glycosaminoglycan concentrations in synovial fluid from the metacarpophalangeal joint of horses. Am J Vet Res 2004, 65, 296-302.

27. Verma P., Dalal K.: ADAMTS-4 and ADAMTS-5: key enzymes in osteoarthritis. J Cell Biochem 2011, 112, 3507-3514.

28. Wang G., Li X., Jiang R., Li Y., Fan X., Zheng Y., Gao L.: Changes in synovial fluid inflammatory mediators and cartilage biomarkers after experimental acute equine synovitis. Bull Vet Inst Pulawy 2015, 59, 129-131.

29. Zheng W., Zhang H., Jin Y., Wang Q., Chen L., Feng Z., Chen H., Wu Y.: Butein inhibits IL-1beta-induced inflammatory response in human osteoarthritis chondrocytes and slows the progression of osteoarthritis in mice. Int Immunopharmacol 2017, 42, 1-10. 